\title{
EFFECTS OF NEW OPACITY ON THE POST-AGB EVOLUTION
}

\author{
M. KATO \\ Department of Astronomy, Keio University, Yokohama 223, Japan \\ and
}

I. HACHISU

Department of Earth Science and Astronomy, University of Tokyo, Tokyo 153, Japan

\begin{abstract}
Using the OPAL opacity, we have calculated the post-AGB evolutions of low mass stars. It is newly found that optically thick wind occurs for $M_{\text {core }}>0.55 M_{\odot}$ and the evolutionary time scale is drastically shortened.
\end{abstract}

Key words: post-AGB evolution, optically thick wind, mass loss, novae

\section{Optically thick winds on $M_{\text {core }}>0.55 M_{\odot}$}

We have examined the effect of the new opacity (Rogers \& Iglesias 1992, private communication) on the post-AGB evolution of low mass stars. The new opacity has a strong peak ( 2 or 3 times larger) around $\log T=5.5$ due to the iron lines compared with the old opacity. The increase in the opacity leads to the decrease in the Eddington luminosity so that we expect strong wind mass loss even for low mass $\left(M_{\text {core }} \sim 0.6 M_{\odot}\right)$ post-AGB stars, which did show no wind mass loss for the old opacity (Kato \& Hachisu 1989, ApJ, 364, 424.).

Figure 1 shows our new results of the post-AGB evolution of low mass stars with the core mass ranging from $0.5 M_{\odot}$ to $1.377 M_{\odot}$. Optically thick wind occurs for $M_{\text {core }}>0.55 M_{\odot}$ while stars with the core mass of $0.5 M_{\odot}$ do not show any wind mass loss mainly because the luminosity does not exceed the Eddington luminosity.

The wind mass loss drastically shortens the time scale of the post-AGB evolution. For example, the evolutionary time from $\log T=4$ to the end point of mass loss in Figure 1 is $12 \mathrm{yr}$ and $24 \mathrm{yr}$ for $0.7 M_{\odot}$ and $0.6 M_{\odot}$, respectively.

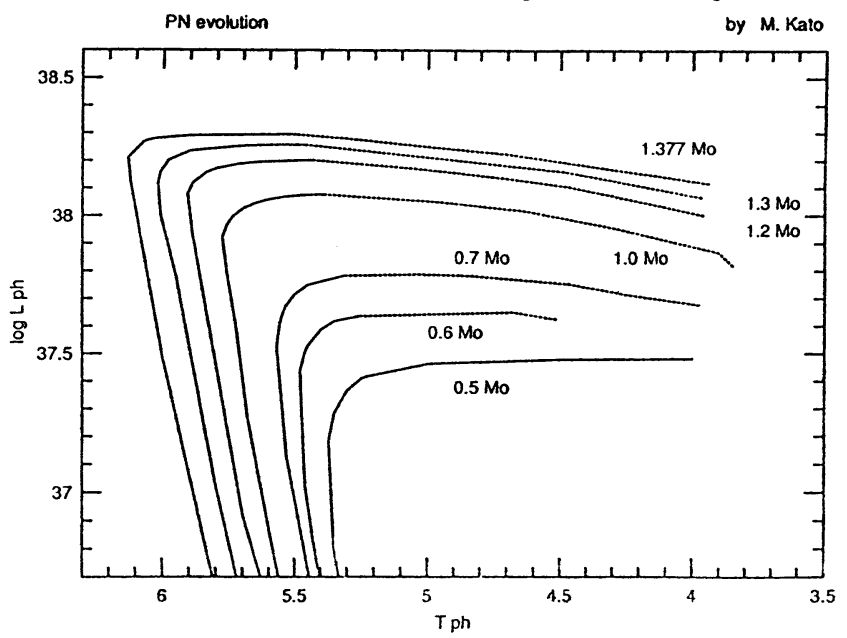

Fig. 1. Theoretical H-R diagram for $M_{\text {core }}=0.5,0.6,0.7,1.0,1.2,1.3$, and $1.37 \mathrm{i} M_{\odot}$. Dashed part of the lines denote the wind mass loss phase. 